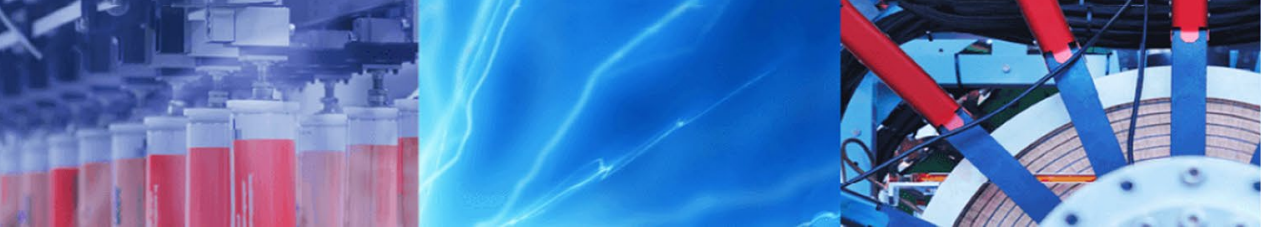

Research Article

\title{
Isolation of secondary metabolites from the mediterranean sponge species; Hemimycale columella and its biological properties
}

\author{
Ilias Marmouzi ${ }^{1}$ (D) Shahira M. Ezzat ${ }^{2,3}$ (1) $\cdot$ Eman Sherien Mostafa ${ }^{3} \cdot$ Meryem El Jemli $^{1,7} \cdot$ Rasha Ali Radwan $^{4}$. \\ My El Abbes Faouzi ${ }^{1}$ - Naoufal Tamsouri ${ }^{5} \cdot$ Mourad Kharbach $^{1,6}$
}

Received: 7 September 2020 / Accepted: 22 December 2020 / Published online: 25 January 2021

(c) The Author(s) $2021 \quad$ OPEN

\begin{abstract}
Despite the richness and biodiversity of invertebrates and algae in the Mediterranean Sea, these organisms are still poorly studied. The objective of our research is the discovery of bioactive lead compounds from the Mediterranean Sea sponge Hemimycale Collumella (HC). HC sponge (189.0 g) was collected from Mdiq costs on the Mediterranean Sea and extracted with methanol to yield $(10 \mathrm{~g})$ which was then subjected to fractionation. A bio-guided protocol was applied through evaluation of 2,2-diphenyl-1-picrylhydrazyl (DPPH) scavenging and Oxygen Radical Absorbance Capacity (ORAC), $\alpha$-amylase, $\beta$-glucosidase, pancreatic lipase inhibition as well as anti-collagenase, anti-elastase, antityrosinase and cytotoxic activity. 2,3-O-Hexahydroxydiphenoyl-( $\alpha / \beta)$-glucose (1) and gentisic acid 2-O- $\beta$-glucoside (2) were isolated from the water fraction, quercetin-3-O- $\beta$-glucopyranoside (3), kaempferol 3-O- $\beta$-glucopyranoside (4) and isorhamnetin 3-O- $\beta$-glucopyranoside (5) from $n$-butanol fraction, gallic acid (6) from ethyl acetate fraction and gallic acid-3-methyl ether (7) from methylene chloride fraction. Compound 5 had the highest DPPH and ORAC activity. Compounds 1-5 had promising lipase inhibition activities which exceeded that of the standard Orlistat, while compounds 1-7 showed anti-tyrosinase activity higher than that of the standard Hydroquinone monomethyl ether. This is the first report for evaluation of the biological activities of 2, 3-O-hexahydroxydiphenoyl-( $\alpha / \beta)$-glucose (1), gentisic acid 2-O- $\beta$-glucoside (2) and gallic acid-3-methyl ether (7).
\end{abstract}

Keywords Hemimycale collumella $\cdot 2,3-O-h e x a h y d r o x y d i p h e n o y l-(\alpha / \beta)$-glucose $\cdot$ Quercetin-3-O- $\beta$-glucopyranoside . Gallic acid - Gallic acid-3-methyl ether

\section{Introduction}

The increasing incidence of health problems caused by certain cancers, drug-resistant pathogenic microbes, parasitic protozoans and fungi have evoked the interest for newer and more effective therapeutic agents. It is acknowledged that marine invertebrates produce bioactive natural products that may be useful for developing new drugs. By exploring untapped geographical sources and/or novel groups of organisms, one can maximize the search for new marine drugs to treat human diseases. Secondary metabolites obtained from marine sponges have

$\triangle$ Shahira M. Ezzat, shahira.ezzat@pharma.cu.edu.eg| ${ }^{1}$ Laboratory of Pharmacology and Toxicology, Biopharmaceutical and Toxicological Analysis Research Team, Faculty of Medicine and Pharmacy, Mohammed V University in Rabat, BP 6203, Rabat, Morocco. ${ }^{2}$ Department of Pharmacognosy, Faculty of Pharmacy, Cairo University, Kasr-el-Aini street, Cairo 11562, Egypt. ${ }^{3}$ Pharmacognosy Department, Faculty of Pharmacy, October University for Modern Sciences and Arts (MSA), Giza 12451, Egypt. ${ }^{4}$ Biochemistry Department, Faculty of Pharmacy, East Kantara Branch, Sinai University, New City El Ismailia 41611, Egypt. ${ }^{5}$ Specialized Center in Zootechny and Marine Aquaculture Engineering, National Institute of Fisheries Research, BP. 31, 93200 M'diq, Morocco. ${ }^{6}$ Department of Analytical Chemistry, Applied Chemometrics and Molecular Modelling, Vrije Universiteit Brussel (VUB), Laarbeeklaan 103, B-1090 Brussels, Belgium. ${ }^{7}$ Present Address: Faculty of Pharmacy, Mohammed VI University of Health Sciences, Casablanca, Morocco. 
been shown to be biologically active compounds with anticancer and antimicrobial activities [1].

From 1963 to 2005, about 24,662 new compounds were isolated and identified from various marine macroor micro-organisms [2]. Among the oldest multicellular invertebrate organisms are marine sponges that belong to Porifera phylum, which have various colors and shapes [3]. More than 10,000 species of marine sponge were authenticated and described [4]. Marine sponges are a very attractive source of bioactive natural products as they yielded a diverse variety of bioactive compounds, among which about 10 bioactive compounds had reached late phases in clinical trials $[5,6]$. This fact demonstrates our interest in marine sponges for drug discovery and development [6]. Marine sponges of phylum Porifera are rich sources of biologically-active compounds, these compounds are biosynthesized by clusters of functional enzyme in the sponges and their associated microorganisms. Each year about 200 new metabolites are reported from sponges $[5,6]$. Among these compounds are the anti-viral drug vidarabine [9- $\beta-D-$ arabinofuranosyladenine (ara-A)], which was effective against herpes simplex encephalitis virus, the beta-carboline alkaloid, manzamine $A$ was proved effectiveness against malaria, tuberculosis and HIV, in addition to the antifungal macrolide compound, lasonolides and the antibacterial psammaplin A [5].

The Mediterranean Sea is acknowledged for its rich biodiversity and high endemism that provide strong motivation to explore the chemical diversity of new interesting metabolites with pharmaceutical applications. However, despite the richness and biodiversity of the Moroccan sea, invertebrates and algae from the seabed are poorly studied. Accordingly, the objective of this research project aims at the discovery of new anti-tumor and bioactive lead compounds from the poorly investigated Moroccan marine sponges collected from the Mediterranean Sea. Members of the genus Hemimycale (Family Hymedesmiidae, Order Poecilosclerida) were reported to have many bioactive secondary metabolites such as guanidine alkaloids $[7,8]$ and hydantoin derivatives which were isolated from $\mathrm{H}$. Arabica obtained from the Red Sea $[9,10]$. Our plant of interest is Hemimycale collumella, the species of Hemimycale, which is widely distributed across the Atlanto-Mediterranean basin. No reports were found concerning its chemical composition and biological activities, so it was deemed of interest for our team to explore its bioactive constituents through a bio-guided fractionation protocol.

\section{Materials and methods}

\subsection{General experimental procedures}

$1 \mathrm{H}-\mathrm{NMR}$ spectra were measured by a Jeol ECA $500 \mathrm{MHz}$ NMR spectrometer, at $500 \mathrm{MHz}$. $1 \mathrm{H}$ chemical shifts $(\delta)$ were measured in ppm, relative to TMS and ${ }^{13} \mathrm{C} N M R$ chemical shifts to DMSO- $d 6$ and converted to TMS scale by adding 39.5. FTESIMS spectra were measured on a Finnigan LTQ-FTMS (Thermo Electron, Bremen, Germany) (Department of Chemistry, Humboldt-Universitat zu Berlin). ORAC experiment was performed on fluorometer, FLUOstar OPTIMA, Franka Ganske, BMG LABTECH, Offenburg, Germany. UV recording were made on a Shimadzu UV-Visible-1601 spectrophotometer. Paper chromatographic analysis was carried out on Whatman No. 1 paper, using solvent systems: (1) $\mathrm{H}_{2} \mathrm{O}$; (2) $6 \%$ acetic acid in water $(6 \% \mathrm{AcOH})$; (3) BAW (n-butanol- acetic acid $-\mathrm{H}_{2} \mathrm{O}, 4: 1: 5$, upper layer). Sephadex LH 20 (Pharmacia, Stockholm, Sweden). All chemicals and reagents for in vitro assays were purchased from (sigma, USA). Plates were purchased from (Mekkawy, Egypt). Spectrophotometric measurements were done on ELX 808 (Bio Tek Instrumental, Italy).

\subsection{Hemimycale columella (HC)}

The marine sponges were collected in March 2014 from Mdiq costs on the Mediterranean Sea by "Institut National de Recherche Halieutique" (Casablanca, Morocco) and identified later at the zoology department in "naturalis biodiversity center" (Leiden, Netherlands) as Hemimycale collumella (RMNH POR 10,012) by Nicole J. de Voogd (National Museum of Natural History).

\subsection{Extraction of hemimycale columella (HC)}

Sponge material was exhaustively extracted with $\mathrm{MeOH}$ $(2 \mathrm{~L} \times 3)$ at room temperature $(\mathrm{RT})$ and the total extracts were concentrated under vacuum at $40{ }^{\circ} \mathrm{C}$ in a rotary evaporator. The $\mathrm{HC}$ collection (189.0 $\mathrm{g}$ wet weight) yielded $10 \mathrm{~g}$ of crude viscous oily methanol extract. The extract was suspended in distilled water $(200 \mathrm{ml})$ and subjected to subsequent liquid - liquid partition afforded n-hexane $(\mathrm{HCH})$, methylene chloride $(\mathrm{HCL}), \mathrm{EtOAc}(\mathrm{HCE}), \mathrm{n}-\mathrm{BuOH}$ $(\mathrm{HCB})$ and residual water fractions (HCW). Each fraction was concentrated under vacuum to yield $72 \mathrm{mg}, 321 \mathrm{mg}$, $467 \mathrm{mg}, 1.367 \mathrm{~g}$ and $1.450 \mathrm{~g}$ of HCH, HCL, HCE, HCB and HCW, respectively. 


\subsection{Radical scavenging activity using DPPH assay}

The estimation was done according to the method of Brand-Williams et al. (1995) [11]. All experiments were carried out in triplicate. Ascorbic acid was used as positive control.

\subsection{Oxygen radical absorbance capacity (ORAC assay)}

The antioxidant capacity in phosphate-buffered saline (10 mM, pH 7.4) were assayed by measuring the time of fluorescein fluorescence decay (Sigma), produced by 2,2'-azobis (2-amidinopropane) dihydrochloride (AAPH) in comparison with the positive control trolox control $[12,13]$.

\subsection{Alpha amylase inhibition assay}

The a-amylase bioassay method was adopted [14] using acarbose as a standard. The starch solution $(0.5 \% \mathrm{w} / \mathrm{v})$ was obtained by stirring potato starch in $20 \mathrm{mmol} / \mathrm{L}$ sodium phosphate buffer $(\mathrm{pH} 7.0)$ and $6.7 \mathrm{mmol} / \mathrm{L}$ sodium chloride. The tested sample was dissolved in the buffer (31.25-1000 $\mu \mathrm{gm} / \mathrm{L})$. The colorimetric reagent was prepared by mixing sodium potassium tartrate solution $(12.0 \mathrm{~g} / 8.0 \mathrm{ml}$ of $2 \mathrm{M} \mathrm{NaOH})$ and $96 \mathrm{mmol} / \mathrm{L}$ of 3,5-dinitro salicylic acid solution. Both control and extracts were mixed with starch solution and left to react with a-amylase solution in alkaline conditions at room temperature. The generated maltose by measuring the absorbance of 3-amino5-nitrosalicylic acid resulted from the reduction of 3,5-dinitrosalicylic acid at $540 \mathrm{~nm}$.

\section{7 $\beta$-Glucosidase inhibition assay}

$1.0 \mathrm{ml}$ of $0.1 \mathrm{M}$ acetate buffer ( $\mathrm{pH} 5.0)$ was mixed with $0.5 \mathrm{ml}$ of $20 \mathrm{mM}(603 \mathrm{mg} p$-nitrophenyl- $\beta$-Dglucopyranoside/100 $\mathrm{ml}$ of $\mathrm{H}_{2} \mathrm{O}$ ) (PNPG) and incubated at $37^{\circ} \mathrm{C}$ for $5 \mathrm{~min}$ then mixed with $0.5 \mathrm{ml}$ of the $\beta$-glucosidase enzyme $(50 \mathrm{mM}$ in Tris- $\mathrm{HCl}$ buffer $\mathrm{pH}$ 7.8 and diluted in $10 \mathrm{mM}$ phosphate buffer $\mathrm{pH} 7.0$ ) and incubated again for $15 \mathrm{~min}$ at $37^{\circ} \mathrm{C}$. $2.0 \mathrm{ml} \mathrm{Na}_{2} \mathrm{CO}_{3}$ solution was used to stop the reaction. The absorbance was measured at $400 \mathrm{~nm}$ [15-17].

\subsection{Pancreatic lipase inhibition assay}

The inhibition of pancreatic lipase was determined as described by [18] using orlistat as a positive control.
$100 \mu \mathrm{L}$ of the substrate 4-nitrophenyl octanoate (NPC) ( $5 \mathrm{mmol} . \mathrm{L}^{-1}$ in DMSO) was mixed with $4 \mathrm{~mL}$ of Tris- $\mathrm{HCl}$ buffer (pH 8.5), $100 \mu \mathrm{L}$ of each tested sample and $100 \mu \mathrm{L}$ of aqueous solution of porcine pancreatic lipase $(1 \mathrm{mg} /$ $\mathrm{mL}$ ). The mixture was incubated at $37^{\circ} \mathrm{C}$ for $25 \mathrm{~min}$. The absorbance was measured at $412 \mathrm{~nm}$.

\subsection{Anti-collagenase assay}

$25 \mu \mathrm{l}$ of collagenase type 1 from Clostridium histolyticum (1 mg/ml), 25 ul tris(hydroxymethyl)-methyl-2-aminoethane sulfonate (TES) buffer $(50 \mathrm{mM})$ with $0.36 \mathrm{mM}$ calcium chloride $(\mathrm{pH} 7.4)$ and test sample or epigallocatechin gallate which was used as a positive standard (EGCG) $\left(1.4 \mathrm{mg} / \mathrm{ml}\right.$ in DMSO) were incubated at $37{ }^{\circ} \mathrm{C}$ for $20 \mathrm{~min}$. $100 \mu \mathrm{l} \mathrm{N}$-[3-(2-furyl)acryloyl]-Leu-Gly-Pro-Ala (FALGPA) was added to the mixtures and kept again for $1 \mathrm{~h}$ at $37^{\circ} \mathrm{C}$. $200 \mu \mathrm{l}$ of a mixture of equal volumes of $200 \mathrm{mM}$ citrate buffer ( $\mathrm{pH} 5)$ and ninhydrin were then added. The mixtures were incubated at $100{ }^{\circ} \mathrm{C}$ for $5 \mathrm{~min}$ then $200 \mu \mathrm{l}$ of $50 \%$ isopropanol were added. The absorbance was measured at $540 \mathrm{~nm}$ [19].

\subsection{Anti-elastase assay}

The anti-elastase activity was assessed in accordance with the method of Kraunsoe et al. [20], with minor modifications. The test samples $(25 \mu \mathrm{l})$ or standard $(1.4 \mathrm{mg} / \mathrm{ml}$ in DMSO) were incubated at $25^{\circ} \mathrm{C}$ for 20 min with $25 \mu \mathrm{l}$ of human leukocyte elastase $(1 \mu \mathrm{g} / \mathrm{ml})$ and $25 \mu \mathrm{l}$ 4-(2-hydroxyethyl)- 1-piperazineethane sulfonic acid (HEPES) buffer (pH 7.5). The substrate N-Methoxysuccinyl-Ala-Ala-ProVal-p-nitroanilide $(1 \mathrm{mM})(100 \mu \mathrm{l})$ was then added and the mixtures were incubated for $40 \mathrm{~min}$ at room temperature. Absorbance was read at $405 \mathrm{~nm}$ using Elafin as a positive control.

\subsection{Anti-tyrosinase assay}

Inhibition of tyrosinase was determined using hydroquinone monomethyl ether as positive standard [21]. Mushroom tyrosinase enzyme (5600 units $/ \mathrm{ml})(80 \mu \mathrm{l})$ was incubated with $80 \mu \mathrm{l}$ of $1 \mathrm{mg} / \mathrm{ml}$ test sample or standard at $37^{\circ} \mathrm{C}$ for $15 \mathrm{~min}$. Then $40 \mu \mathrm{l}$ of $L-3,4$-dihydroxyphenylalanine (L-DOPA) was added and incubated at $37^{\circ} \mathrm{C}$ for $30 \mathrm{~min}$. Absorbance of the formed Dopachrome is measured at $475 \mathrm{~nm}$.

\subsection{Neutral red uptake assay (NRU)}

Non-tumorigenic $\mathrm{HaCaT}$ cells, bladder carcinoma cell line (5637 cells), breast carcinoma (MCF7), hepatocellular 
carcinoma (Huh7) were obtained from the Vaccera (Giza, Egypt). Cytotoxicity of the fractions and isolates against the four cell lines was carried out using the neutral red uptake (NRU) assay [22]. Measurement was done at $450 \mathrm{~nm}$ in a plate reader and $\mathrm{IC}_{50}$ values were determined and expressed in mean $\pm S D$. All samples were tested in triplicate using Etoposide as positive control.

\subsection{Statistical analysis}

All the assays were carried out in triplicate. The results are expressed as mean values and standard deviations (SDs). The $\mathrm{IC}_{50}$ (concentration necessary for $50 \%$ inhibition of enzyme activity) was calculated by constructing a linear regression curve showing extracts concentrations percentage inhibition on the $y$-axis[23]. All analysis were done using the SPSS v. 22.0 (IBM, Chicago, USA). Microsoft Excel 2010 was used for graph construction.

\subsection{Isolation and identification of the major phenolic compounds from the bioactive fractions}

HCW (1 g) was fractionated using Sephadex LH-20 column (50 gm) and elution was done by $\mathrm{H}_{2} \mathrm{O} / \mathrm{H}_{2} \mathrm{O}-\mathrm{MeOH}$ mixtures where fractions (I and II) were obtained and investigated using 2D-PC (two dimensional paper chromatography). Fraction I (eluted with $10 \% \mathrm{MeOH}$ in water, $320 \mathrm{mg}$ ) was then purified over Sephadex $\mathrm{LH}-20(20 \mathrm{~g})$ using $\mathrm{H}_{2} \mathrm{O}$ for elution to yield compound $\mathbf{1}(20 \mathrm{mg}$ ) in a pure from. Compound 2 (15 mg) was separated by purification of fraction II (eluted with $20 \%, \mathrm{MeOH}$ in water, $730 \mathrm{mg}$ ) over Sephadex LH-20 $(25 \times 1 \mathrm{~cm}, 20 \mathrm{~g})$ using $\mathrm{H}_{2} \mathrm{O}$ as eluent.

$\mathrm{HCB}(1 \mathrm{~g})$ was fractionated as for HCW to yield three fractions (III-V). Fraction IV yield Compound 3 (32 mg) (elution with $30 \% \mathrm{MeOH}$ in water, $210 \mathrm{mg}$ ) using preparative $\mathrm{PC}$ and $6 \% \mathrm{AcOH}$ (solvent). Fraction $\mathrm{V}$ yields compound $\mathbf{4}$ (25 mg) and 5 (30 mg) (eluted with $60 \% \mathrm{MeOH}$ in water, $435 \mathrm{mg}$ ) by preparative PC using $6 \% \mathrm{AcOH}$ (solvent). HCE fraction (300 mg) was fractionated over Sephadex $\mathrm{LH}-20$, using $\mathrm{MeOH}-\mathrm{H}_{2} \mathrm{O}$ mixtures of different polarities. The fraction eluted with $80 \% \mathrm{MeOH}$ in water $(200 \mathrm{mg})$ from which pure samples of compound 6 (40 mg) was isolated using preparative $P C$ and BAW as solvent system.

HCL (100 mg) was purified over several Sephadex $\mathrm{LH}-20$ ( $20 \mathrm{~g}$ ) using MeOH for elution to yield compound 7 (18 mg).

\subsubsection{Compound (1)}

Rf values: $0.66\left(\mathrm{H}_{2} \mathrm{O}\right), 0.78$ (6\% $\mathrm{AcOH}$ in water), 0.22 (BAW), $\mathrm{UV} \lambda_{\max }(\mathrm{nm})$ in $\mathrm{MeOH}: 259$ (inflection).

${ }^{1} \mathrm{H}$ - NMR spectral data (DMSO-d6) $\delta$ (ppm):
Glucose moiety: $5.32\left(\mathrm{~d}, J=3.5 \mathrm{~Hz}, \alpha-\mathrm{H}-1^{\prime \prime}\right), 4.90(J=8 \mathrm{~Hz}$, $\left.\beta-\mathrm{H}-1^{\prime \prime}\right), 4.64\left(\mathrm{dd}, J=8 \& 7.5, \beta-\mathrm{H}-2^{\prime \prime}\right), 4.83(\mathrm{dd}, J=3.5 \& 8 \mathrm{~Hz}$, $\left.a-\mathrm{H}-2^{\prime \prime}\right), 4.93\left(\mathrm{t}, J=7.5 \mathrm{~Hz}, \beta-\mathrm{H}-3^{\prime \prime}\right), 5.25\left(\mathrm{t}, J=8 \mathrm{~Hz}, a-\mathrm{H}-3^{\prime \prime}\right)$, 3.3-3.8 (m, H-4"-H-6").Hexahydroxydiphenoyl (HHDP) in $a-\& \beta$-anomers: $6.53,6.54,6.61,6.62\left(\mathrm{~s}, \mathrm{H}-3\right.$ \& $\left.\mathrm{H}-3^{\prime}\right)$.

${ }^{13} \mathrm{C}-N M R$ Spectral Data (DMSO- $\left.d 6\right) \delta$ (ppm): $a$-glucose moiety: $90.50\left(C-1^{\prime \prime}\right), 72.06\left(C-2^{\prime \prime}\right), 77.43\left(C-3^{\prime \prime}\right), 67.24$ (C-4"), $77.01\left(C-5^{\prime \prime}\right), 61.05\left(C-6^{\prime \prime}\right) . \beta$-glucose moiety:93.77 $\left(C-1^{\prime \prime}\right), 74.72\left(C-2^{\prime \prime}\right), 79.71\left(C-3^{\prime \prime}\right), 67.53\left(C-4^{\prime \prime}\right), 77.01\left(C-5^{\prime \prime}\right)$, 61.05(C-6"). (HHDP) in $a-\& \beta$-anomers: $113.93,113.77$ (C-1 \& $\left.C-1^{\prime}\right), 125.67,125.93,126.17\left(C-2 \& C-2^{\prime}\right), 106.60,106.84$ (C-3 \& C-3'), 143.64, 144.57 (C-4, C-4'and C-6 \& C-6'), 135.71, $135.72\left(C-5 \& C-5^{\prime}\right), 168.88,169.02,169.55(C=0)$.

\subsubsection{Compound (2)}

Rf values: $0.35\left(\mathrm{H}_{2} \mathrm{O}\right), 0.48$ (6\% $\mathrm{AcOH}$ in water), 0.19 (BAW), UV $\lambda_{\text {max }}(\mathrm{nm})$ in $\mathrm{MeOH}: 282,393$.

${ }^{1} \mathrm{H}$ - NMR spectral data (DMSO-d6) $\delta$ (ppm):

Sugar moiety:4.71 $(1 \mathrm{H}, d, J=7 \mathrm{~Hz}, \mathrm{H}-1)$. Aglycone:7.20 $\left(1 \mathrm{H}, d, J=2.5 \mathrm{~Hz}, \mathrm{H}-6^{\prime}\right), 6.71\left(1 \mathrm{H}, d d, J=2.5,8.4 \mathrm{~Hz}, \mathrm{H}-4^{\prime}\right)$, $6.52\left(1 \mathrm{H}, d, J=8.4 \mathrm{~Hz}, \mathrm{H}-3^{\prime}\right)$.

${ }^{13} \mathrm{C}-\mathrm{NMR}$ Spectral Data (DMSO-d6) $\delta$ (ppm):

$\beta$-glucose moiety: 96.8 (C-1), 72.9 (C-2), 76.6 (C-3), 69.6(C-4), 76.4 (C-5), 61.0 (C-6).

Aglycone: $116.5\left(C-1^{\prime}\right), 148.2\left(C-2^{\prime}\right), 115.0\left(C-3^{\prime}\right), 121$ $\left(C-4^{\prime}\right), 154.3\left(C-5^{\prime}\right), 115.0\left(C-6^{\prime}\right), 171.9(C=0)$.

\subsubsection{Compound (3)}

Rf values: $0.8\left(\mathrm{H}_{2} \mathrm{O}\right), 0.44$ (6\% AcOH in water), 0.32 (BAW).), UV $\lambda_{\text {max }}(\mathrm{nm})$ in MeOH:256, 265 sh., 358; + NaOMe 268, 327, $403+\mathrm{NaOAc} 273,323,387 ;+\mathrm{NaOAc}-\mathrm{H}_{3} \mathrm{BO}_{3} 262,377 ;+\mathrm{AlCl}_{3}$ $273,430$.

${ }^{1} \mathrm{H}-\mathrm{NMR}$ spectral data (DMSO-d6) $\delta$ (ppm):

Quercetin moeity: $7.68\left(2 \mathrm{H}, \mathrm{m}, \mathrm{H}-2^{\prime}\right.$ and $\left.\mathrm{H}-6^{\prime}\right), 6.87(1 \mathrm{H}, d$, $\left.J=8.5 \mathrm{~Hz}, \mathrm{H}-5^{\prime}\right), 6.39(\mathrm{IH}, d, J=2 \mathrm{~Hz}, \mathrm{H}-8), 6.17(\mathrm{IH}, d, J=2 \mathrm{~Hz}$, H-6).Glucoside moiety: $5.53(1 \mathrm{H}, d, J=8 \mathrm{~Hz}, \mathrm{H}-1 "), 3.2-3.9$ (glucoside protons overlapped with hydroxyl and water protons).

${ }^{13} \mathrm{C}-\mathrm{NMR}$ Spectral Data (DMSO-d6) $\delta$ (ppm):

Quercetin moeity: 156.6 (C-2), 133.80 (C-3), 177.9 (C-4), 161.71 (C-5), 99.20(C-6), 164.8 (C-7), 95.62(C-8), 156.91 (C-9), $104.42(C-10), 122.10\left(C-1^{\prime}\right), 115.70\left(C-2^{\prime}\right), 145.30$ (C-3'), $149.0\left(\mathrm{C}-4^{\prime}\right), 116.7\left(\mathrm{C}-5^{\prime}\right), 122.10\left(\mathrm{C}-6^{\prime}\right) ; \beta$-glucose moiety: 101.32(C-1"), 74.60(C-2"), 76.05(C-3"), 70.40(C-4"), 77.70(C-5"), $61.50\left(\mathrm{C}-6^{\prime \prime}\right)$.

\subsubsection{Compound (4)}

Rf values: $0.24\left(\mathrm{H}_{2} \mathrm{O}\right), 0.47(6 \% \mathrm{AcOH}$ in water), 0.76 (BAW). UV $\lambda_{\max }(\mathrm{nm})$ in MeOH: 266, 345 + NaOMe: 271, 
$376,+\mathrm{NaOAC}: 270,346,+\mathrm{NaOAC}+\mathrm{H}_{3} \mathrm{BO}_{3}: 270,346$ sh., $405,+\mathrm{Al} \mathrm{Cl}_{3}: 268,340$ sh., 385.

${ }^{1} \mathrm{H}$ - NMR spectral data (DMSO-d6) $\delta(\mathrm{ppm})$ :

kaempferol moiety: $6.186(\mathrm{~d}, J=2.5 \mathrm{~Hz}, \mathrm{H}-6), 6.38(\mathrm{~d}$, $J=2.5 \mathrm{~Hz}, \mathrm{H}-8), 7.7\left(\mathrm{~d}, J=2.5, \mathrm{H}-2^{\prime}, \mathrm{H}-6^{\prime}\right), 6.89(\mathrm{~d}, J=8 \mathrm{~Hz}, \mathrm{H}-$

$\left.3^{\prime}, \mathrm{H}-5^{\prime}\right) . \beta$-glucose moiety: $5.26\left(\mathrm{~d}, J=8 \mathrm{~Hz}, \mathrm{H}-1^{\prime \prime}\right), 3.4-4.0$ (m, H-2"- H-6").

${ }^{13} \mathrm{C}-\mathrm{NMR}$ Spectral Data (DMSO-d6) $\delta$ (ppm):

Kaempferol moiety: 156.93 (C-2), 134.20 (C-3), 177.93 (C-4), 160.28 (C-5), 99.33 (C-6), 165.20 (C-7), 94.24 (C-8), 157.86 (C-9), $104.16(C-10), 121.20\left(C-1^{\prime}\right), 131.28\left(C-2^{\prime}, C-6^{\prime}\right)$, $115.36\left(C-3^{\prime}, C-5^{\prime}\right), 161.33\left(C-4^{\prime}\right) . \beta$-glucose moiety: 103.23 $\left(C-1^{\prime \prime}\right), 74.33\left(C-2^{\prime \prime}\right), 76.49\left(C-3^{\prime \prime}\right), 69.71\left(C-4^{\prime \prime}\right), 76.69\left(C-5^{\prime \prime}\right)$, $61.18\left(C-6^{\prime \prime}\right)$.

\subsubsection{Compound (5)}

Rf values: $0.11\left(\mathrm{H}_{2} \mathrm{O}\right), 0.12$ (6\% AcOH in water), 0.52 (BAW). UV $\lambda_{\max }(\mathrm{nm})$ in MeOH: 256, 300 sh., 354, + NaOMe: 275, 325 sh., 408, + NaOAC: $275,323,371,+\mathrm{NaOAC}+\mathrm{H}_{3} \mathrm{BO}_{3}$ : 276, 295 sh., $355+\mathrm{AlCl}_{3}: 269,365,403$.

${ }^{1} \mathrm{H}$ - NMR spectral data (DMSO- $\left.d 6\right) \delta(\mathrm{ppm})$ :

Isorhamnetin moiety: $6.21(\mathrm{~d}, J=1.6 \mathrm{~Hz}, \mathrm{H}-6), 6.42(\mathrm{~d}$, $J=1.6 \mathrm{~Hz}, \mathrm{H}-8), 3.83\left(\mathrm{~s}, \mathrm{OCH}_{3}\right), 7.93\left(\mathrm{~d}, J=1.6 \mathrm{~Hz}, \mathrm{H}-2^{\prime}\right)$, $6.92\left(\mathrm{~d}, J=8.4 \mathrm{~Hz}, \mathrm{H}-5^{\prime}\right), 7.55$ (dd, $J=1.6$ and $8.4 \mathrm{~Hz}, \mathrm{H}-6^{\prime}$ ). $\beta$-glucose moiety: $5.57\left(\mathrm{~d}, J=8 \mathrm{~Hz}, \mathrm{H}-1^{\prime \prime}\right), 3.0-3.8(\mathrm{~m}$, $\left.\mathrm{H}-2^{\prime \prime}-\mathrm{H}-6^{\prime \prime}\right)$.

${ }^{13} \mathrm{C}-\mathrm{NMR}$ Spectral Data (DMSO-d6) $\delta$ (ppm):

Isorhamnetin moiety: 156.30 (C-2), 133.20 (C-3), 177.30 (C-4), 161.1 (C-5), 98.70 (C-6), 164.31 (C-7), 93.61 (C-8), $156.21(\mathrm{C}-9), 103.90(\mathrm{C}-10), 55.60\left(\mathrm{OCH}_{3}\right), 120.90\left(\mathrm{C}-1^{\prime}\right)$, 113.40 (C-2'), $148.41\left(\mathrm{C}-3^{\prime}\right), 149.31\left(\mathrm{C}-4^{\prime}\right), 115.10\left(\mathrm{C}-5^{\prime}\right)$, $122.0\left(C-6^{\prime}\right)$. $\beta$-glucose moiety: $100.8\left(C-1^{\prime \prime}\right), 74.21\left(C-2^{\prime \prime}\right)$, $76.30\left(C-3^{\prime \prime}\right), 70.40\left(C-4^{\prime \prime}\right), 77.30\left(C-5^{\prime \prime}\right), 60.51\left(C-6^{\prime \prime}\right)$.

\subsubsection{Compound (6)}

$R_{f}$ values: $44\left(\mathrm{H}_{2} \mathrm{O}\right), 0.55$ (6\% AcOH in water), 0.72 (BAW); UV Spectral Data $\lambda_{\max }(\mathrm{nm}): 272$.

${ }^{1} \mathrm{H}$ - NMR Spectral Data (DMSO-d6) $\delta$ (ppm): 6.98 (S, H-2 and $\mathrm{H}-6)$.

\subsubsection{Compound (7)}

Rf values: $0.0 .50\left(\mathrm{H}_{2} \mathrm{O}\right), 0.53(6 \% \mathrm{AcOH}$ in water), 0.84 (BAW), UV $\lambda_{\max }(\mathrm{nm})$ in $\mathrm{MeOH} ; 273$.

${ }^{1} \mathrm{H}$ - NMR spectral data (DMSO-d6) $\delta(\mathrm{ppm}): 7.24(d$, $J=2.5 \mathrm{~Hz}, \mathrm{H}-2), 7.15(d, J=2.5 \mathrm{~Hz}, \mathrm{H}-6), 3.8(\mathrm{~s}, 3 \mathrm{H}, 3-\mathrm{OMe})$.

${ }^{13} \mathrm{C}-\mathrm{NMR}$ Spectral Data (DMSO-d6) $\delta$ (ppm):120.90 (C-1), 105.21 (C-2), 148.61 (C-3), 139.50 (C-4), 145.90 (C-5), 111.26 $(\mathrm{C}-6), 167.32(\mathrm{C}=0), 56.32(3-\mathrm{OMe})$.

\section{Results and discussion}

The ocean covers nearly $75 \%$ of the Earth's surface, which offers a huge biodiversity and interesting source of natural products. Marine sponges play an indispensable ecological role in preserving a stable marine ecosystems by factor of their symbiosis with other organisms [24]. Currently more than 8,500 sponge species are confirmed valid from more than 11,000 species, which could be classified in four different classes including Calcarea (calcareous sponges), Hexactinellida (glass sponges), Sclerospongiae (coralline sponges) and Demospongiae (horny sponges, siliceous sponges or common sponges) [25], 25 orders, 680 genera and 128 families [26]. Diversity of marine sponges and especially their secondary metabolites are considered as a gold mine for discovering bioactive components with great therapeutic, cosmetic and nutritional importance. In fact, the sponge biomass culture may conduct alternative compounds for new drug development in pharmaceutical aspects [25]. However, the lack of appropriate ethno-medical history, technical problems in collection or culturing and the variety of marine species organisms may directly affect the development of marine-derived products as beneficial agents.

Marine sponges are aquatic invertebrates, which are a rich source of bioactive metabolites (i.e. amino acids, alkaloids, aliphatics, sterols, macrolides, glycosides, ketones, lipids, macrolides, peptides, nucleosides, porphyrins, fatty acids, esters, steroids, terpenes, terpenoids, terpenoids) and enzymes [27]. Marine organisms contain secondary bioactive metabolites, which were proved to have medicinal, biological and pharmacological effects including antioxidant, antiviral, anti-malarial, anti-tuberculosis, antibacterial, anti-inflammatory, immunosuppressive, antifungal, antiviral, anticancer, antitumor, anticoagulant, neurosuppressor and enzyme inhibitory agents [28]. However, the search for those secondary metabolites includes three steps: chemical structure identification, kind of pharmacological potential and biological mechanism of action.

Determining the antioxidant potential by measuring antiradical scavenging has been widely applied to indicate the antioxidant properties in plants, food, beverages and marine products. However, antioxidant potential of marine sponges and its metabolites are poorly investigated [29-31].

Antioxidant refers to any substance that inhibits a free radical reaction. These substances possess importance as health promoters in the treatment of cardiovascular diseases, atherosclerosis and anti-cancer ageing [32]. Few studies concerning antioxidant activity of marine sponges were reported, such as antioxidant and cytotoxic activities of some Philippian marine sponges [33]; antioxidant 
potential of Petrosia sp., Oceanapia ramsayi, Clathria sp., Ancorina cerebrum and Haliclona fascigera sponges [34]. In the current study, the antioxidant activity of the Mediterranean sponge Hemimycale columella different fractions ( $n$-hexane $(\mathrm{HCH})$, methylene chloride $(\mathrm{HCL})$, ethyl acetate (HCE), n-butanol (HCB) and water (HCW) fractions) were evaluated using two methods 2,2-diphenyl-1-picrylhydrazyl (DPPH) scavenging and oxygen radical absorbance capacity (ORAC). The antioxidant capacities issued by DPPH showed a highly inhibitory potential of HCB, HCE and HCW (Table 1). HCB, followed by HCE and HCW fractions exhibited the highest ORAC antioxidant capacity which exceeded that of the standard Trolox. The activity of $\mathrm{HCL}$ extract is comparable to the activity of Trolox. The obtained results were promising and indicated that $H$. columella sponge exhibited an antioxidant ability.

The antidiabetic potential of marine sponge has been poorly investigated, where evaluation of the a-amylase inhibition activity of 24 Antarctic marine sponge was reported [35]; a-amylase inhibitory of Antarctic marine sponges (Porifera) and a-glucosidase inhibition of marine sponges collected in Mauritius waters [36]; $\alpha$-glucosidase Inhibitors from the Penares schulzei marine Sponge [37]; a-glucosidase inhibition of 1,4-dideoxy-1,4-imino-d-arabinitol isolated from two marine sponges collected from Western Australia [38]; $\beta$-glucosidase inhibition of fungal endophyte of the marine sponge Latrunculia corticata [39].

Table 1 the antioxidant activity of the extracts and isolated compounds

\begin{tabular}{lll}
\hline The tested sample & \multicolumn{2}{l}{$\mathrm{ED}_{50}(\mu \mathrm{g} / \mathrm{ml})$} \\
\cline { 2 - 3 } & $\mathrm{DPPH}$ & $\mathrm{ORAC}$ \\
\hline $\mathrm{HCH}$ & $36.15 \pm 1.77$ & $35.91 \pm 2.15$ \\
$\mathrm{HCL}$ & $27.18 \pm 2.91$ & $26.89 \pm 2.37$ \\
$\mathrm{HCE}$ & $10 \pm 0.88$ & $10 \pm 0.13$ \\
$\mathrm{HCB}$ & $8 \pm 2.15$ & $7.81 \pm 1.18$ \\
Trolox & - & $27.0 \pm 13.41$ \\
Vit. C & $1.83 \pm 1.41$ & - \\
The tested compound & $\mathrm{ED}_{50}(\mu \mathrm{M})$ & $\mathrm{DPPH}$ \\
Compound 1 & $40.456 \pm 1.35$ & $39.419 \pm 1.34$ \\
Compound 2 & $71.46 \pm 3.67$ & $69.25 \pm 3.12$ \\
Compound 3 & $81.83 \pm 2.13$ & $79.676 \pm 2.59$ \\
Compound 4 & $73.599 \pm 2.58$ & $27.261 \pm 2.55$ \\
Compound 5 & $25.54 \pm 1.22$ & $26.65 \pm 1.32$ \\
Compound 6 & $455.267 \pm 1.22$ & $452.62 \pm 2.13$ \\
Compound 7 & $233.505 \pm 1.45$ & $217.214 \pm 2.66$ \\
Trolox & - & $107.87 \pm 13.41$ \\
Vit. C & $10.391 \pm 1.41$ & - \\
\hline
\end{tabular}

Results are given as mean values $\pm S D$ of $n=3$ independent experiments
$a$-Amylase, $a$ - and $\beta$-glucosidase inhibition is an effective approach for diabetic care [40]. Thus, isolation of glucosidase and amylase inhibitors from natural sources such as marine organisms can represent a promising strategy in drug discovery [40]. Few sponges were subjected to evaluation of their $\alpha$-amylase, $\alpha$ - and $\beta$-glucosidase inhibition activity such as some Antarctic marine sponges (Porifera) from Mauritius waters [36], Penares schulzei marine Sponge [37],1,4-dideoxy-1,4-imino-d-arabinitol isolated from two marine sponges collected from Western Australia [38]; endophyte of the marine sponge Latrunculia corticata [39]. Our findings showed that HCL (85\%) exhibited the highest a-amylase inhibitory activity, followed by HCB (68\%), HCW (68\%), HCE (65\%) and HCH (21\%) extract. HCB only exhibited $\beta$-glucosidase inhibitory effect (64\%), while the other fractions were inactive (Table 2).Our results revealed a good anti-diabetic potential of $H$. columella sponge fractions regarding the $a$-amylase inhibition. Moreover, the pancreatic lipase formed by pancreatic acinar-cells is responsible for $50 \%-70 \%$ hydrolysis of dietary triacylglycerols into monoacylglycerol and fatty acids; its inhibition contribute in treatment of both diabetes and obesity [41]. Our results highlighted a potent pancreatic lipase of $H$. columella sponge five fractions (Table 2) where HCW, $\mathrm{HCH}, \mathrm{HCL}$ and HCE had about $91 \%$ inhibitory effect. They showed $\mathrm{IC}_{50}$ values that were even lower than that of the standard Orlistat.

Collagenase and elastase are two enzymes responsible for dehydration and wrinkle formation on the skin surface. Secondary metabolites isolated from natural products including flavonoids, tocopherols, phenolics and tannins have demonstrated anti-collagenase and anti-elastase activities [42]. The anti-aging properties of plant extracts are generally attributed to their antioxidants, which reduce free radical scavenging and provide skin protection [43]. Some metabolites such as mycosporine-like amino-acids isolated from marine organisms were reported to possess in vitro anti-aging and wound-healing properties including inhibition of collagenase [44]. Three tiglic acid-containing cyclodepsipeptides (largamides) metabolites isolated from marine cyanobacterium Lyngbya confervoides showed an elastase inhibitory activity [45]. Secondary metabolites such as mycosporine-like amino acids isolated from marine red algae Porphyra sp. and Palmaria palmata exhibited collagenase inhibitory potential [46].

Tyrosinase is an important enzyme which catalyzes melanin synthesis in melanocytes $[47,48]$. Currently, tyrosinase inhibition is a promising approach for the development of skin whitening and cosmetic products. In our study, the collagenase, elastase inhibitory effects of $\mathrm{H}$. columella sponge fractions were also examined. The results (Table 3), showed that HCE and $\mathrm{HCH}$ had about a $75 \%$ inhibition effect on the collagenase enzyme. HCE

SN Applied Sciences 
Table 2 a-Amylase, $\beta$-glucosidase and pancreatic lipase inhibition effect of the extracts and compounds (Mean \pm S.D.) and their $\mathrm{IC}_{50}($ $\mathrm{IC}_{50} \pm$ S.D.)

\begin{tabular}{|c|c|c|c|c|c|c|}
\hline \multirow[t]{2}{*}{ The tested sample } & \multicolumn{2}{|c|}{ Inhibition of a-amylase } & \multicolumn{2}{|c|}{ Inhibition of $\beta$-glucosidase } & \multicolumn{2}{|c|}{ Inhibition of pancreatic lipase } \\
\hline & $\begin{array}{l}\% \text { Inhibition of at } \\
600 \mu \mathrm{g} / \mathrm{ml}\end{array}$ & $\begin{array}{l}\mathrm{IC}_{50} \\
\mu \mathrm{g} / \mathrm{ml}\end{array}$ & $\begin{array}{l}\% \text { Inhibition of at } \\
600 \mu \mathrm{g} / \mathrm{ml}\end{array}$ & $\begin{array}{l}\mathrm{IC}_{50} \\
\mu \mathrm{g} / \mathrm{ml}\end{array}$ & $\begin{array}{l}\% \text { Inhibition at } 100 \mu \mathrm{g} / \\
\mathrm{ml}\end{array}$ & $\begin{array}{l}\mathrm{IC}_{50} \\
\mu \mathrm{g} / \mathrm{ml}\end{array}$ \\
\hline $\mathrm{HCH}$ & $21.4 \pm 6.4$ & $1061.1 \pm 11.8$ & - & - & $92.9 \pm 5.9$ & $12.6 \pm 6.4$ \\
\hline $\mathrm{HCL}$ & $85.1 \pm 7.1$ & $181.67 \pm 9.8$ & - & - & $92.2 \pm 5.1$ & $13.79 \pm 4.6$ \\
\hline $\mathrm{HCE}$ & $64.7 \pm 5.7$ & $321.83 \pm 7.9$ & - & - & $91.01 \pm 5.6$ & $11.69 \pm 6.9$ \\
\hline $\mathrm{HCB}$ & $67.7 \pm 5.6$ & $312.16 \pm 12.4$ & $64 \pm 5.9$ & $343.17 \pm 8.8$ & $85.4 \pm 5.9$ & $15.64 \pm 5.1$ \\
\hline $\mathrm{HCW}$ & $67.5 \pm 5.3$ & $294.7 \pm 8.5$ & - & - & $94.5 \pm 6.4$ & $9.926 \pm 5.8$ \\
\hline Acarbose & $94.1 \pm 7.2$ & $230.85 \pm 2.45$ & - & - & - & - \\
\hline 1-Deoxynojrimycin & - & - & $98.3 \pm 0.4$ & $27.72 \pm 0.02$ & - & - \\
\hline Orlistat & - & - & - & - & $92 \pm 6.4$ & $28.96 \pm 6.4$ \\
\hline \multirow[t]{2}{*}{ The tested sample } & \multicolumn{2}{|c|}{ Inhibition of a-amylase } & \multicolumn{2}{|c|}{ Inhibition of $\beta$-glucosidase } & \multicolumn{2}{|c|}{ Inhibition of pancreatic lipase } \\
\hline & $\begin{array}{l}\% \text { Inhibition of at } \\
600 \mu \mathrm{g} / \mathrm{ml}\end{array}$ & $\begin{array}{l}\mathrm{IC}_{50} \\
(\mu \mathrm{M})\end{array}$ & $\begin{array}{l}\% \text { Inhibition of at } \\
600 \mu \mathrm{g} / \mathrm{ml}\end{array}$ & $\begin{array}{l}\mathrm{IC}_{50} \\
(\mu \mathrm{M})\end{array}$ & $\begin{array}{l}\% \text { Inhibition at } 100 \mu \mathrm{g} / \\
\mathrm{ml}\end{array}$ & $\begin{array}{l}\mathrm{IC}_{50} \\
(\mu \mathrm{M})\end{array}$ \\
\hline Compound 1 & $80 \pm 5.1$ & $571.16 \pm 10.1$ & - & - & $93.4 \pm 5.4$ & $16.57 \pm 2.43$ \\
\hline Compound 2 & $72.4 \pm 6.2$ & $1113.64 \pm 8.6$ & - & - & $94 \pm 5.1$ & $35.79 \pm 3.1$ \\
\hline Compound 3 & $32.4 \pm 6.5$ & $2111.18 \pm 14.3$ & - & - & $92.4 \pm 4.1$ & $25.41 \pm 3.9$ \\
\hline Compound 4 & $59.2 \pm 5.37$ & $1137.88 \pm 9.6$ & - & - & $84.4 \pm 5.2$ & $27.72 \pm 3.95$ \\
\hline Compound 5 & $25.3 \pm 5.8$ & $2086.12 \pm 11.5$ & - & - & $85.9 \pm 4.7$ & $25.15 \pm 4.2$ \\
\hline Compound 6 & $76.2 \pm 5.9$ & $2314.24 \pm 12.3$ & - & - & $93.8 \pm 4.8$ & $66.54 \pm 4.2$ \\
\hline Compound 7 & $52 \pm 8.7$ & $3157.15 \pm 10.8$ & - & - & $83 \pm 4.5$ & $91.12 \pm 4.7$ \\
\hline Acarbose & $94.1 \pm 7.2$ & $357.57 \pm 2.45$ & - & - & - & - \\
\hline 1-Deoxynojrimycin & - & - & $98.3 \pm 0.4$ & $169.884 \pm 0.02$ & - & - \\
\hline Orlistat & - & - & - & - & $92 \pm 6.4$ & $58.41 \pm 6.4$ \\
\hline
\end{tabular}

Results are given as mean values $\pm S D$ of $n=3$ independent experiments

exhibited the highest collagenase inhibition activity. The elastase inhibitory capacity of the five fractions (Table 3 ) was higher than on collagenase, which might contribute to their antiwrinkle properties. The tyrosinase inhibition effects of $H$. columella sponge fractions were also summarized in Table 3. Both HCL and HCE showed a higher tyrosinase inhibitory potential (81\%) than the three other fractions with $\mathrm{IC}_{50}$ values lower than that of the standard Hydroquinone monomethyl ether (149.9 \pm 5.8 and $155.6 \pm 7.3 \mu \mathrm{g} / \mathrm{ml}$, respectively). This study revealed that the different fractions of $H$. columella sponge exhibited high or satisfactory anti-collagenase, anti- elastase and tyrosinase inhibitory effects.

Marine sponges derived metabolites including terpenes, alkaloids, peptides, aromatics, lactones, steroids and miscellaneous compounds are evidenced cytotoxic and anticancer agents [49]. However, the anti-cancer drugs from marine products as sources of natural metabolites is still in research development [50]. The cytotoxicity potential of the five fractions of $\mathrm{H}$. columella sponge was assessed against four tumorous human cell lines: bladder carcinoma cell line (5637 cells), hepatocellular carcinoma (Huh7), breast carcinoma (MCF7) and immortalized human keratinocytes ( $\mathrm{HaCaT}$ cells) while etoposide was used as a positive control. The highest cytotoxic potential against bladder carcinoma cell line were obtained for $\mathrm{HCE}\left(\mathrm{IC} \mathrm{C}_{50}=8.52 \mu \mathrm{g} / \mathrm{ml}\right)$ and $\mathrm{HCB}\left(\mathrm{IC}_{50}=11.52 \mu \mathrm{g} / \mathrm{ml}\right)$. On the other hand, $\mathrm{HCB}\left(\mathrm{IC}_{50}=2.98 \mu \mathrm{g} / \mathrm{ml}\right)$ revealed a good inhibitory activity against hepatocellular carcinoma. Moreover, the five fractions showed high safety on the immortalized human keratinocytes normal cells. Based upon the obtained results, $\mathrm{HCL}, \mathrm{HCE}, \mathrm{HCB}$ and $\mathrm{HCW}$ were investigated for the isolation of their major compounds.

The isolated compounds (1-7) showed chromatographic, UV absorption and hydrolytic data identical with those reported for 2,3-O-hexahydroxydiphenoyl- $(\alpha / \beta)$ glucose (1) [51], gentisic acid 2-O- $\beta$-glucoside (2) [52] which were isolated from $\mathrm{HCW}$, quercetin-3-O- $\beta$-glucopyranoside (3) [53], kaempferol 3-O- $\beta$-glucopyranoside (4) [54], isorhamnetin 3-O- $\beta$-glucopyranoside (5) [55] from $\mathrm{HCB}$; gallic acid (6) [56] from HCE and gallic acid-3-methyl ether (7) $[57,58]$ from HCL. Structures of the isolated compounds are shown in Fig. 1.

Compound (1), (20 mg) off-white amorphous powder, appeared as a blue spot under short UV light on PC and gives deep blue color upon spraying with $\mathrm{FeCl}_{3}$. It gives red 
Table 3 Collagenase, elastase and tyrosinase inhibition of different extracts and isolated compounds (Mean \pm S.D.) and their IC ${ }_{50}\left({ }^{\prime} C_{50} \pm\right.$ S.D.)

\begin{tabular}{|c|c|c|c|c|c|c|}
\hline \multirow[t]{2}{*}{ The tested Sample } & \multicolumn{2}{|c|}{ Inhibition of collagenase } & \multicolumn{2}{|c|}{ Inhibition of elastase } & \multicolumn{2}{|c|}{ Inhibition of tyrosinase } \\
\hline & $\begin{array}{l}\% \text { Inhibition at } \\
500 \mu \mathrm{g} / \mathrm{ml}\end{array}$ & $\begin{array}{l}\mathrm{IC}_{50} \\
\mu \mathrm{g} / \mathrm{ml}\end{array}$ & $\begin{array}{l}\% \text { Inhibition at } \\
300 \mu \mathrm{g} / \mathrm{ml}\end{array}$ & & $\begin{array}{l}\% \text { Inhibition at } \\
500 \mu \mathrm{g} / \mathrm{ml}\end{array}$ & $\begin{array}{l}\mathrm{IC}_{50} \\
\mu \mathrm{g} / \mathrm{ml}\end{array}$ \\
\hline $\mathrm{HCH}$ & $75.1 \pm 7.65$ & $200.4 \pm 6.6$ & $87.7 \pm 4.8$ & $106.09 \pm 5.4$ & $75.8 \pm 6.1$ & $188.9 \pm 6.9$ \\
\hline $\mathrm{HCL}$ & $71.2 \pm 6.6$ & $217.19 \pm 7.5$ & $90.2 \pm 5.9$ & $100.6 \pm 4.7$ & $80.6 \pm 7.6$ & $149.9 \pm 5.8$ \\
\hline $\mathrm{HCE}$ & $75.2 \pm 4.9$ & $196.5 \pm 5.1$ & $88.2 \pm 4.7$ & $102.2 \pm 7.5$ & $81.2 \pm 5.4$ & $155.6 \pm 7.3$ \\
\hline $\mathrm{HCB}$ & $71 \pm 5.7$ & $224.5 \pm 5.6$ & $87.8 \pm 7.6$ & $105.7 \pm 7.1$ & $72 \pm 5.8$ & $207.8 \pm 5.3$ \\
\hline $\mathrm{HCW}$ & $72 \pm 7.4$ & $224.11 \pm 7.4$ & $88.1 \pm 7.2$ & $111.94 \pm 6.9$ & $64 \pm 6.3$ & $323.75 \pm 7.8$ \\
\hline EGCG & $89.10 \pm 2.7$ & $112.12 \pm 9.4$ & - & - & - & - \\
\hline Elafin & - & - & $59.5 \pm 7.1$ & $250.0 \pm 12.6$ & - & - \\
\hline $\begin{array}{l}\text { Hydroquinone mono- } \\
\text { methyl ether }\end{array}$ & - & - & - & - & $55.2 \pm 2.2$ & $330.0 \pm 4.1$ \\
\hline \multirow[t]{2}{*}{ The tested Sample } & \multicolumn{2}{|c|}{ Inhibition of collagenase } & \multicolumn{2}{|c|}{ Inhibition of elastase } & \multicolumn{2}{|c|}{ Inhibition of tyrosinase } \\
\hline & $\begin{array}{l}\% \text { Inhibition at } \\
500 \mu \mathrm{g} / \mathrm{ml}\end{array}$ & $\begin{array}{l}\mathrm{IC}_{50} \\
(\mu \mathrm{M})\end{array}$ & $\begin{array}{l}\% \text { Inhibition at } \\
300 \mu \mathrm{g} / \mathrm{ml}\end{array}$ & $\begin{array}{l}\mathrm{IC}_{50} \\
(\mu \mathrm{M})\end{array}$ & $\begin{array}{l}\% \text { Inhibition at } \\
500 \mu \mathrm{g} / \mathrm{ml}\end{array}$ & $\begin{array}{l}\mathrm{IC}_{50} \\
(\mu \mathrm{M})\end{array}$ \\
\hline Compound 1 & $56 \pm 4.6$ & $1237.34 \pm 6.5$ & $79.8 \pm 7.5$ & $369.295 \pm 4.3$ & $74.2 \pm 6.9$ & $382.99 \pm 7.2$ \\
\hline Compound 2 & $69 \pm 6.7$ & $1129.261 \pm 6.3$ & $77.1 \pm 6.9$ & $564.41 \pm 5.5$ & $75 \pm 5.8$ & $574.84 \pm 4.6$ \\
\hline Compound 3 & $71.5 \pm 7.3$ & $563.116 \pm 4.9$ & $93 \pm 6.1$ & $222.232 \pm 7.6$ & $76 \pm 5.3$ & $459.959 \pm 4.6$ \\
\hline Compound 4 & $65.1 \pm 6.3$ & $857.75 \pm 6.8$ & $87.9 \pm 5.2$ & $228.61 \pm 6.4$ & $72 \pm 7.3$ & $421.8995 \pm 7.1$ \\
\hline Compound 5 & $70.2 \pm 5.8$ & $710.97 \pm 6.4$ & $81.2 \pm 4.1$ & $320.65 \pm 4.7$ & $72.8 \pm 6.2$ & $385.66 \pm 4.3$ \\
\hline Compound 6 & $58.5 \pm 6.1$ & $2671.64 \pm 8.6$ & $90.4 \pm 4.6$ & $626.62 \pm 5.9$ & $81.1 \pm 7.3$ & $889.078 \pm 5.7$ \\
\hline Compound 7 & $74 \pm 6.7$ & $1152.1 \pm 6.75$ & $92.5 \pm 5.5$ & $566.929 \pm 4.6$ & $62 \pm 5.6$ & $1510.72 \pm 6.7$ \\
\hline EGCG & $89.10 \pm 2.7$ & $244.605 \pm 9.4$ & - & - & - & - \\
\hline Elafin & - & - & $59.5 \pm 7.1$ & $41.67 \pm 12.6$ & - & - \\
\hline $\begin{array}{l}\text { Hydroquinone mono- } \\
\text { methyl ether }\end{array}$ & - & - & - & - & $55.2 \pm 2.2$ & $2658.35 \pm 4.1$ \\
\hline
\end{tabular}

Results are given as mean values \pm SD of $n=3$ independent experiments

with nitrous acid specific for ellagitannins. In the $1 \mathrm{H}-\mathrm{NMR}$ spectrum of (DMSO- $d_{6}$, room temperature), it showed two doublets appearing at $\delta 5.32(J=3.5 \mathrm{~Hz})$ and $4.90(J=8 \mathrm{~Hz})$ of the $\alpha$ - and $\beta$-anomeric glucose protons, respectively, confirming the free anomeric hydroxyl group. The $\mathrm{H}-2^{\prime \prime}$ glucose proton appears at $\delta \mathrm{ppm} 4.64(\mathrm{dd}, J=8$ and $7.5 \mathrm{~Hz})$ and at 4.83 (dd, $J=3.5$ and $8 \mathrm{~Hz}$ ), in both $\beta$ - and $\alpha$ - anomers, respectively. Also, the $\mathrm{H}-3$ " glucose protons in the $\beta$ - and $\alpha$-anomers appeared at $\delta \mathrm{ppm} 4.93(\mathrm{t}, J=7.5 \mathrm{~Hz})$ and at $5.25(\mathrm{t}, J=8 \mathrm{~Hz})$, respectively. The downfield shifts of $\mathrm{H}-2$ " and $\mathrm{H}-3$ ", confirmed the attachment of HHDP moiety at C-2" and C-3" of glucose. The HHDP moiety appeared as two pairs of singlets in the aromatic region at $\delta 6.62$, $6.61,6.54$ and 6.53 , referring to the $\mathrm{H}-3$ and $\mathrm{H}-3^{\prime}$ in both anomers. The remaining glucose proton appeared as a multiplet from $\delta 3.3$ to $3.8 \mathrm{ppm}$. The ${ }^{13} \mathrm{C}$-NMR spectrum (DMSO- $d_{6}$, room temperature) finally confirmed the structure of (1) and the measured chemical shift values of the glucose carbon resonances proved that the sugar core is in the pyranose form.

Compound (2), (20 mg), amorphous off- white powder. It appeared as a blue spot under short UV light on PC and gives dirty blue color upon spraying with $\mathrm{FeCl}_{3}$. Normal acid hydrolysis $\left(2 \mathrm{~N}\right.$ aqueous $\mathrm{HCL}, 3 \mathrm{~h}, 100^{\circ} \mathrm{C}$ ) yields gentisic acid and glucose (CoPC). In ${ }^{1} \mathrm{H}-\mathrm{NMR}$ (DMSO- $d_{6}$, room temperature) the $\beta$-glucose anomeric proton appeared at $\delta 4.71(\mathrm{~d}, J=7 \mathrm{~Hz})$ whose germinal $\mathrm{OH}$ is involved in the acetal linkage and the attachment of gentisic acid and glucose moiety confirmed by ${ }^{13} \mathrm{C}-\mathrm{NMR}$ (DMSO- $d_{6}$, room temperature) as seven chemical shift values appeared for gentisic acid in which the C-2 hydroxyl group is substituted by the anomeric glucose carbon at $\delta 96.80$ (C-1). This was concluded from the upfield shift of the aromatic C-2 carbon all in comparison with the corresponding chemical shifts of free gentisic acid [52]. Also, $\beta$-glucose configuration was confirmed from the $C-I$ chemical shift.

Compound (3), (32 mg) a yellowish brown amorphous powder. It appeared as a dark purple spot under short UV light on $\mathrm{PC}$ and turned orange when fumed with ammonia. Normal acid hydrolysis $\left(2 \mathrm{~N} \mathrm{HCl}, 3 \mathrm{~h}, 100^{\circ} \mathrm{C}\right.$ ) yields quercetin and glucose (CoPC). Also, upon enzymatic hydrolysis with $\beta$-glucosidase, quercetin was released (CoPC). In ${ }^{1} \mathrm{H}$-NMR (DMSO- $d_{6}$, room temperature) the $\beta$-glucose anomeric proton appeared $\delta 5.53 \mathrm{ppm}(\mathrm{d}$, $J=8 \mathrm{~Hz}$ ) and other resonance chemical shifts were agreed with the suggested structure of both sugar and flavonoid 


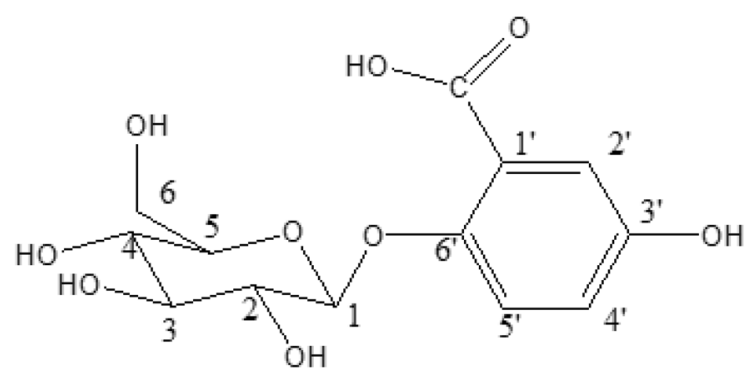

\section{Compound 2}

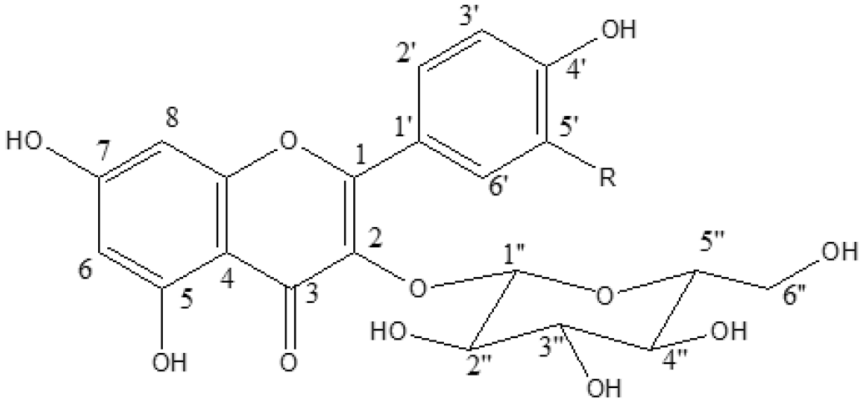

Compound 3: $\mathrm{R}=\mathrm{OH}$

Compound 4: $\mathrm{R}=\mathrm{H}$

Compound 5: $\mathrm{R}=\mathrm{OCH}_{3}$

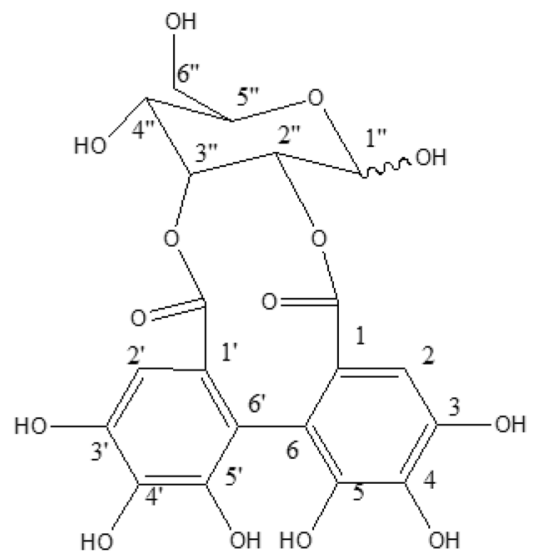

Compound 1<smiles>[R9]c1cc(C(=O)O)cc(O)c1O</smiles>

Compound 6: $\mathbf{R}=\mathbf{H}$ Compound 7: $\mathrm{R}=\mathrm{CH}_{3}$

Fig. 1 Structures of the isolated compounds

moieties [53]. In ${ }^{13} \mathrm{C}$-NMR (DMSO-d6, room temperature) the presence of one $\beta$-glucose moiety confirmed from the anomeric carbon resonance at $\delta 101.32 \mathrm{ppm}$ and from the chemical shift values of the remaining sugar resonances in the region from $\delta 61.50$ to $76.05 \mathrm{ppm}$. The flavonoid moiety resonances were in agreement with the corresponding published signals [53].

Compound (4), (25 mg) a brown amorphous powder. It appeared as a dark purple spot under short UV light on $P C$ and turned yellow when fumed with ammonia. Normal acid hydrolysis $\left(2 \mathrm{~N} \mathrm{HCl}, 3 \mathrm{~h}, 100^{\circ} \mathrm{C}\right.$ ) yields kaempferol and glucose (CoPC). Also, upon enzymatic hydrolysis with $\beta$-glucosidase, kaempferol was released (CoPC). In ${ }^{1} \mathrm{H}$-NMR (DMSO- $d_{6}$, room temperature) the $\beta$-glucose anomeric proton appeared $\delta 5.26 \mathrm{ppm}(\mathrm{d}, J=8 \mathrm{~Hz})$ and other resonance chemical shifts were agreed with the suggested structure of both sugar and flavonoid moiety [54]. In ${ }^{13} \mathrm{C}$-NMR (DMSO- $d_{6}$, room temperature) the presence of one $\beta$-glucose moiety confirmed from the anomeric carbon resonance at $\delta 103.23 \mathrm{ppm}$ and from the chemical shift values of the remaining sugar resonances in the region from $\delta 61.18$ to $76.69 \mathrm{ppm}$. The flavonoid moiety resonances were in agreement with the corresponding published signals [53].

Compound (5), (30 mg) a yellow amorphous powder. It appeared as a dark purple spot under short UV light on $\mathrm{PC}$ and turned lemon yellow when fumed with ammonia. Normal acid hydrolysis $\left(2 \mathrm{~N} \mathrm{HCl}, 3 \mathrm{~h}, 100^{\circ} \mathrm{C}\right)$ yields isorhamnetin and glucose (COPc and UV). In ${ }^{1} \mathrm{H}-\mathrm{NMR}$ (DMSO$d_{6}$, room temperature) the $\beta$-glucose anomeric proton appeared $\delta 5.57 \mathrm{ppm}(\mathrm{d}, J=8 \mathrm{~Hz})$. The flavonol moiety $\mathrm{H}-6$ and $\mathrm{H}-8$ appeared at $\delta 6.21 \mathrm{ppm}(\mathrm{d}, J=1.6 \mathrm{~Hz})$ and $\delta 6.42 \mathrm{ppm}\left(\mathrm{d}, J=1.6 \mathrm{~Hz}\right.$ ), respectively. Also, $\mathrm{H}-5^{\prime}$ and $\mathrm{H}-6^{\prime}$ appears at $\delta$ ppm $6.92\left(\mathrm{~d}, J=8.4 \mathrm{~Hz}, \mathrm{H}-5^{\prime}\right), 7.55$ (dd, $J=8.4 \& 1.6 \mathrm{~Hz}, \mathrm{H}-6^{\prime}$ ) while the methoxy group appears at $\delta 3.83 \mathrm{ppm}$. Furthermore, ${ }^{13} \mathrm{C}-\mathrm{NMR}$ analysis (DMSO$d_{6}$, room temperature) confirmed the structure of (5) in comparison with the corresponding published data [55].

Compound (6), ( $40 \mathrm{mg}$ ) a white powder. It appeared as a blue spot under short UV light on PC which gave a blue colour with $\mathrm{FeCl}_{3}$. Structure confirmation was carried out by CoPc and further by ${ }^{1} \mathrm{H}-\mathrm{NMR}$ analysis $\left(D M S O-d_{6}\right.$, room temperature). The spectrum showed one singlet signal in 
Table 4 The cytotoxic activity of the extracts and isolated compounds

\begin{tabular}{|c|c|c|c|c|}
\hline \multirow[t]{2}{*}{ The tested samples } & \multicolumn{4}{|l|}{$\begin{array}{l}\text { Cytotoxicity } \\
{\left[\mathrm{IC}_{50}(\mu \mathrm{g} / \mathrm{mL})\right]}\end{array}$} \\
\hline & $\begin{array}{l}\text { Bladder carci- } \\
\text { noma cell line } \\
\text { ( } 5637 \text { cells) }\end{array}$ & $\begin{array}{l}\text { Hepatocellualr carcinoma } \\
\text { (Huh 7) }\end{array}$ & Breast cancer cell line (MCF7) & $\begin{array}{l}\text { Immortalized human keratinocytes } \\
\text { ( } \mathrm{HaCaT} \text { cells) }\end{array}$ \\
\hline $\mathrm{HCH}$ & $32.10 \pm 0.16$ & $9.10 \pm 2.11$ & $10.8 \pm 0.69$ & $410 \pm 6.17$ \\
\hline $\mathrm{HCL}$ & $28.33 \pm 1.32$ & $7.11 \pm 0.12$ & $9.10 \pm 0.17$ & $379 \pm 11.12$ \\
\hline $\mathrm{HCE}$ & $8.52 \pm 0.21$ & $5.10 \pm 1.11$ & $5.33 \pm 1.27$ & $520 \pm 3.83$ \\
\hline $\mathrm{HCB}$ & $11.52 \pm 1.11$ & $2.98 \pm 0.12$ & $3.15 \pm 1.22$ & $569 \pm 9.11$ \\
\hline $\mathrm{HCW}$ & $22.51 \pm 0.18$ & $5.32 \pm 1.21$ & $8.81 \pm 3.51$ & $460 \pm 4.57$ \\
\hline Etoposide & $9.40 \pm 3.34$ & $1.52 \pm 0.15$ & $10.90 \pm 0.24$ & $388 \pm 7.87$ \\
\hline \multirow[t]{2}{*}{ The tested samples } & \multicolumn{4}{|l|}{$\begin{array}{l}\text { Cytotoxicity } \\
{\left[\mathrm{IC}_{50}(\mu \mathrm{g} / \mathrm{mL})\right]}\end{array}$} \\
\hline & $\begin{array}{l}\text { Bladder car- } \\
\text { cinoma cell } \\
\text { line (5637 } \\
\text { cells) }\end{array}$ & $\begin{array}{l}\text { Hepatocellualr carcinoma } \\
\text { (Huh 7) }\end{array}$ & Breast carcinoma cell line (MCF7) & $\begin{array}{l}\text { Immortalized human keratinocytes } \\
\text { ( } \mathrm{HaCaT} \text { cells) }\end{array}$ \\
\hline Compound 1 & $12.45 \pm 1.85$ & $10.37 \pm 2.41$ & $6.66 \pm 1.73$ & $220.98 \pm 10.22$ \\
\hline Compound 2 & $22.134 \pm 0.89$ & $34.05 \pm 1.87$ & $54.765 \pm 2.22$ & $948.587 \pm 4.56$ \\
\hline Compound 3 & $27.801 \pm$ & $12.081 \pm 1.05$ & $13.35 \pm 1.11$ & $925.966 \pm 7.76$ \\
\hline Compound 4 & $38.026 \pm 2.31$ & $18.69 \pm 3.60$ & $10.73 \pm 2.88$ & $838.56 \pm 10.41$ \\
\hline Compound 5 & $63.148 \pm 1.78$ & $23.45 \pm 1.06$ & $17.18 \pm 2.6$ & $1168.77 \pm 12.10$ \\
\hline Compound 6 & $29.39 \pm 4.77$ & $111.686 \pm 2.45$ & $65.31 \pm 1.33$ & $587.82 \pm 3.55$ \\
\hline Compound 7 & $239.18 \pm 0.61$ & $88.623 \pm 2.13$ & $82.54 \pm 3.12$ & $2172.14 \pm 6.33$ \\
\hline Etoposide & $15.97 \pm 3.34$ & $2.58 \pm 0.15$ & $18.52 \pm 0.24$ & $659.19 \pm 7.87$ \\
\hline
\end{tabular}

Results are given as mean values $\pm S D$ of $n=3$ independent experiments

the aromatic region at $\delta 6.98 \mathrm{ppm}$ for the two equivalent $\mathrm{H}-2$ and $\mathrm{H}-6$ protons [56].

Compound (7), (18 mg) an off-white powder. It appeared as a blue spot under short UV light on PC which gave a blue colour with $\mathrm{FeCl}_{3}$. Structure confirmation was carried out by COPC and further by ${ }^{1} \mathrm{H}-\mathrm{NMR}$ analysis (DMSO- $d_{6}$, room temperature). The spectrum showed two doublet signals in the aromatic region at $\delta$ $7.15 \mathrm{ppm}$ and $\delta 7.24 \mathrm{ppm}$ for the two $\mathrm{H}-2$ and $\mathrm{H}-6$ protons and a singlet at $\delta 3.80 \mathrm{ppm}$ for the methoxy group [56]. The ${ }^{13} \mathrm{C}$-NMR spectrum (DMSO- $d_{6}$, room temperature) confirmed the achieved structure [56].

The isolated compounds were also subjected to evaluation of their in vitro activities, compound 5 , followed by 1 and 2 , showed good radical scavenging activity among the tested compounds using DPPH (Table 1). The radical scavenging potential of compound 5 was comparable with that of HCW, while compounds 4 and 5 indicated the highest ORAC antioxidant potential which were stronger than that of the standard Trolox. The isolated compounds had no inhibitory effect on the $\beta$-glucosidase. Meanwhile, the a-amylase inhibitory capacity was highest for compound 1 $(80 \%)$ and $2(72.4 \%)$. Compounds (1-5) showed significant lipase inhibition activity, where they had $\mathrm{IC}_{50}$ values lower than that of Orlistat (Table 2). Moreover, at $100 \mu \mathrm{g} / \mathrm{ml}$, compound 1, 2, 3 and 6 exhibited pancreatic lipase inhibition potential above $92 \%$, while compound 3, 5 and 6 had inhibitory activity of more than $83 \%$. Compound $3(71.5 \%)$ and compound 7 (74\%) showed the highest collagenase inhibition activity, but with $\mathrm{IC}_{50}$ values higher than that of EGCG (Table 3). Concerning the elastase inhibition activity, compound 3,4, 6 and 7 revealed an elastase potential of more than $85 \%$. These results were in accordance with the reported data about quercetin-3-O- $\beta$-glucopyranoside (3) and kaempferol 3-O- $\beta$-glucopyranoside (4) which can be used as valuable agents in cosmetics due to their important chemical characteristics and their ability to possess skin protective effect against UV radiation [59]. Meanwhile, compounds 1-7 showed strong tyrosinase inhibitory activity with IC50 values lower than the standard Hydroquinone monomethyl ether. Gallic acid was reported to significantly inhibit both melanin synthesis and tyrosinase activity in a dose- and time-dependent manner [60]. Compound 1 exhibited the highest cytotoxic potentials against bladder carcinoma cell line (5637 cells) and hepatocellular carcinoma (Huh7) (Table 4). Moreover, compounds 1,3,4,5 had promising anticancer activity against the tested breast cancer cell line (MCF7). On the other hand, the isolated 
compounds except compounds 1 and 6 showed a good safety margin as they had high $\mathrm{IC}_{50}$ against immortalized human keratinocytes, respectively (Table 4). These findings demonstrated a good cytotoxic potential and selectivity of the isolated compounds against different types of cancer cell lines.

Our work presented the initial evaluation of the biological activities of 2, 3-O-hexahydroxydiphenoyl-( $\alpha / \beta)$ glucose (1), gentisic acid 2-O- $\beta$-glucoside (2) and gallic acid-3-methyl ether (7) whose ORAC activities were comparable to that of the standard Trolox. It is also the first study to report the effectiveness of the three compounds as pancreatic lipase and tyrosinase inhibitors as they showed $\mathrm{IC}_{50}$ lower than the standard Orlistat and Hydroquinone monomethyl ether, respectively. 2 , 3-O-hexahydroxydiphenoyl-( $\alpha / \beta)$-glucose (1) and gentisic acid 2-O- $\beta$-glucoside (2) were proved to be promising cytotoxic compounds against Bladder carcinoma cell line (5637 cells), hepatocellular carcinoma (Huh7) and Breast cancer cell line (MCF7) where their IC $C_{50}$ were somewhat comparable to that of the standard Etoposide. It is worthy also to mention that our study is the first report about the collagenase, elastase, tyrosinase and lipase inhibiting activity as well as the cytotoxic activity of isorhamnetin 3-O- $\beta$-glucopyranoside (5).

\section{Conclusion}

The present study is a deep investigation of the biological activities of marine sponges from the Mediterranean. Seven secondary metabolites were isolated, characterized and their biological properties were highlighted. To the best of our knowledge, the present results are the first one to establish antioxidant, antidiabetic, collagenase, elastase, tyrosinase and cytotoxic effects of $H$. columella sponge and its metabolites, especially 2, 3-O-hexahydroxydiphenoyl$(\alpha / \beta)$-glucose (1), gentisic acid 2-O- $\beta$-glucoside (2) and gallic acid-3-methyl ether (7). The data showed that $H$. columella sponge can provide appreciated resources for new pharmaceutical based molecules.

Acknowledgements The authors would like thank Dr. Nicole J. de Voogd (Naturalis Biodiversity Center, Netherlands) for sponge identification and Dr. Sherif S. Ebada (Mutah University, Jordan) for help and support. We are thankful to Kevin Patrick Schnur (University of Chicago at illinois, USA) for English editing.

Funding This study was not funded.

\section{Compliance with ethical standards}

Conflict of interest The authors declare no conficts of interest.
Human and animal rights No human and animal experiments were performed in this study.

Open Access This article is licensed under a Creative Commons Attribution 4.0 International License, which permits use, sharing, adaptation, distribution and reproduction in any medium or format, as long as you give appropriate credit to the original author(s) and the source, provide a link to the Creative Commons licence, and indicate if changes were made. The images or other third party material in this article are included in the article's Creative Commons licence, unless indicated otherwise in a credit line to the material. If material is not included in the article's Creative Commons licence and your intended use is not permitted by statutory regulation or exceeds the permitted use, you will need to obtain permission directly from the copyright holder. To view a copy of this licence, visit http://creativecommons .org/licenses/by/4.0/.

\section{References}

1. Senthilkumar K, Ramajayam G, Venkatesan J, Kim S-K, Ahn B-C (2016) Marine Sponge-Derived Antiangiogenic Compounds for Cancer Therapeutics In Marine Sponges Chemicobiological and Biomedical Applications. Springer. 4:305-314

2. Blun J, Cop B, Keyzers R, Munro M, Prinsep M (2015) Marine natural products. Natural Product Reports 32(1):116-211

3. Fattorusso E, Gerwick WH, Taglialatela-Scafati O (2012) Handbook of marine natural products. Springer, NY

4. Van Soest RW, Boury-Esnault N, Vacelet J, Dohrmann M, Erpenbeck D, De Voogd NJ, Santodomingo N, Vanhoorne B, Kelly M, Hooper JN (2012) Global diversity of sponges (Porifera). PLoS ONE 7(4):e35105

5. Laport M, Santos O, Muricy G (2009) Marine sponges: potential sources of new antimicrobial drugs. Curr Pharm Biotechnol 10(1):86-105

6. Mayer AM, Glaser KB, Cuevas C, Jacobs RS, Kem W, Little RD, Mclntosh JM, Newman DJ, Potts BC, Shuster DE (2010) The odyssey of marine pharmaceuticals: a current pipeline perspective. Trends Pharmacol Sci 31(6):255-265

7. Ohtani I, Kusumi T, Kakisawa H, Kashman Y, Hirsh S (1992) Structure and chemical properties of ptilomycalin A. J Am Chem Soc 114(22):8472-8479

8. Kashman Y, Hirsh S, McConnell OJ, Ohtani I, Kusumi T, Kakisawa H (1989) Ptilomycalin A: a novel polycyclic guanidine alkaloid of marine origin. J Am Chem Soc 111(24):8925-8926

9. Foye WO (2008) Foye's principles of medicinal chemistry. Lippincott Williams \& Wilkins, Philadelphia

10. Youssef DT, Shaala LA, Alshali KZ (2015) Bioactive hydantoin alkaloids from the Red Sea marine sponge Hemimycale arabica. Marine drugs 13(11):6609-6619

11. Mishra K, Ojha H, Chaudhury NK (2012) Estimation of antiradical properties of antioxidants using DPPH assay: A critical review and results. Food Chem 130(4):1036-1043. https://doi. org/10.1016/j.foodchem.2011.07.127

12. Brand-Williams W, Cuvelier ME, Berset C (1995) Use of a free radical method to evaluate antioxidant activity. LWT - Food Science and Technology 28(1):25-30. https://doi.org/10.1016/S0023 $-6438(95) 80008-5$

13. Lucas-Abellan C, Mercader-Ros MT, Zafrilla MP, Fortea MI, Gabaldon JA, Nunez-Delicado E (2008) ORAC-fluorescein assay to determine the oxygen radical absorbance capacity of resveratrol complexed in cyclodextrins. Journal of agricultural and food chemistry 56(6):2254-2259. https://doi.org/10.1021/jf0731088 
14. Miller GL (1959) Use of dinitrosalicylic acid reagent for determination of reducing sugar. Anal Chem 31:426-428

15. Grover AK, Macmurchie DD, Cushley RJ (1977) Characteristics of $\beta$-Glucosidase from almond BiochimBiophysActa 482:98

16. Hash JH, King KW (1958) Some properties of an aryl- $\beta$ glucosidase from culture filtrates of myrothecium verrucaria*. JBiolChem 232:395

17. Heyworth R, Walker PG (1962) Almond-emulsin $\beta$-d-glucosidase and $\beta$-d-galactosidase BiochemJ 83:331-335

18. Conforti F, Perri V, Menichini F, Marrelli M, Uzunov D, Statti GA, al. e, (2012) Wild Mediterranean dietary plants as inhibitors of pancreatic lipase. Phytother Res 26:600-604

19. Thring TS, Hili P, Naughton DP (2009) Anti-collagenase, antielastase and anti-oxidant activities of extracts from 21 plants. BMC ComplementAltern Med 9:27

20. Kraunsoe JAE, Claridge TDW, G. L, (1996) Inhibition of human leukocyte and porcine pancreatic elastase by homologues of bovine pancreatic trypsin inhibitor. Biochemistry 35:9090-9096

21. Rauniyar R, Talkad M, Sahoo S, Singh A, Harlalka P (2014) AntiTyrosinase Activity of Stachytarpheta Cayennensis in Vitro. International Journal of Innovative Research in Science, Engineering and Technology. 3(7):14259-14266

22. Amarowicz R, Pegg RB (2013) Inhibition of proliferation of human carcinoma cell lines by phenolic compounds from a bearberry-leaf crude extract and its fractions. Journal of Functional Foods 5(2):660-667. https://doi.org/10.1016/j. jff.2013.01.009

23. Chiocchio I, Mandrone M, Sanna C, Maxia A, Tacchini M, Poli F (2018) Screening of a hundred plant extracts as tyrosinase and elastase inhibitors, two enzymatic targets of cosmetic interest. Ind Crops Prod 122:498-505

24. Pallela R, Ehrlich H (2016) Marine sponges: Chemicobiological and biomedical applications. Springer, India

25. Santhanam R, Ramesh S, Sunilson AJ (2018) Marine Sponges: Biology and Pharmaceutical Aspects. CRC Press, Biology and Ecology of Pharmaceutical Marine Sponges. https://doi. org/10.1201/9781351132473-2

26. Van Soest RW, Boury-Esnault N, Vacelet J, Dohrmann M, Erpenbeck D, De Voogd NJ, Santodomingo N, Vanhoorne B, Kelly M, Hooper JNJPo, (2012) Global diversity of sponges (Porifera). PLoS ONE 7(4):e35105

27. Heydari H, Gözcelioğlu B, Konuklugil BJRoNP, (2019) Biodiversity and Secondary Metabolites of Marine Sponges from Turkey. ACG publication. 13(5):367-378

28. Parte S, Sirisha V, D'Souza J (2017) Biotechnological applications of marine enzymes from algae, bacteria, fungi and sponges.In Advances in food and nutrition research. Elsevier. $80: 75-106$

29. Utkina N, Makarchenko A, Shchelokova O, Virovaya MJConc (2004) Antioxidant activity of phenolic metabolites from marine sponges. 40 (4);373-377

30. Balakrishnan D, Kandasamy D, Nithyanand PJIJCTR (2014) A review on antioxidant activity of marine organisms. Science Alert. 6(7):3431-3436

31. Takamatsu S, Hodges TW, Rajbhandari I, Gerwick WH, Hamann MT, Nagle DGJJonp, (2003) Marine natural products as novel antioxidant prototypes. J Nat Prod. 66(5):605-608

32. Chairman K, Singh AR, Alagumuthu GJAPJoTD (2012) Cytotoxic and antioxidant activity of selected marine sponges. 2 (3):234-238

33. Rivera A, Uy MJJoC, (2012) In vitro antioxidant and cytotoxic activities of some marine sponges collected off Misamis Oriental Coast. Philippines 9(1):354-358

34. Francisco JT, Uy MMJAJoB, Sciences L, (2016) Toxicity and Antioxidant Potential Screening of Extracts from Five Marine
Sponges Collected off Zamboanga Peninsula, Philippines. Asian Journal of Biological and Life Sciences. 5(3):233-236

35. Berne $S$, Kalauz M, Lapat M, Savin L, Janussen D, Kersken D, Avguštin JA, Jokhadar ŠZ, Jaklič D, Gunde-Cimerman NJPB (2016) Screening of the Antarctic marine sponges (Porifera) as a source of bioactive compounds. Diversity. 39(5):947-959

36. Ramanjooloo A, Cresteil T, Lebrasse C, Beedessee G, Oogarah $P$, van Soest RW, Marie DEJNpr, (2015) a-Glucosidase inhibitory activity of marine sponges collected in Mauritius waters. Nat Prod Res. 29(4):383-387

37. Takada K, Uehara T, Nakao Y, Matsunaga S, van Soest RW, Fusetani NJJotACS, (2004) Schulzeines A-C, new a-glucosidase inhibitors from the marine sponge Penares schulzei. J. Am. Chem. Soc. 126(1):187-193

38. Saludes JP, Lievens SC, Molinski TFJJonp, (2007) Occurrence of the a-glucosidase inhibitor 1, 4-dideoxy-1, 4-imino-D-arabinitol and related iminopentitols in marine sponges. J. Nat. Prod. 70(3):436-438

39. El-Bondkly AM, El-Gendy MMJAVL (2012) Cellulase production from agricultural residues by recombinant fusant strain of a fungal endophyte of the marine sponge Latrunculia corticata for production of ethanol. Antonie Van Leeuwenhoek 101(2):331-346

40. Lauritano C, lanora AJMd (2016) Marine organisms with antidiabetes properties. Mar Drugs. 14(12):220

41. Liang L-F, Wang T, Cai Y-S, He W-F, Sun P, Li Y-F, Huang Q, Taglialatela-Scafati O, Wang H-Y (2014) Guo Y-WJEjomc. Brominated polyunsaturated lipids from the Chinese sponge Xestospongia testudinaria as a new class of pancreatic lipase inhibitors 79:290-297

42. Thring TS, Hili P, Naughton DPJBc, medicine a, (2009) Anti-collagenase, anti-elastase and anti-oxidant activities of extracts from 21 plants. BMC Complementary and Alternative Medicine. $9(1): 27$

43. Lephart EDJARR (2016) Skin aging and oxidative stress: Equol's anti-aging effects via biochemical and molecular mechanisms. Ageing Res Rev. 31:36-54

44. Orfanoudaki M, Hartmann A, Alilou M, Gelbrich T, Planchenault P, Derbré S, Schinkovitz A, Richomme P, Hensel A, Ganzera MJMd (2020) Absolute Configuration of Mycosporine-Like Amino Acids. Their Wound Healing Properties and In Vitro Anti-Aging Effects 18(1):35

45. Matthew S, Paul VJ, Luesch HJPm, (2009) Largamides A-C, tiglic acid-containing cyclodepsipeptides with elastase-inhibitory activity from the marine cyanobacterium Lyngbya confervoides. Planta Med. 75(05):528-533

46. Hartmann A, Gostner J, Fuchs JE, Chaita E, Aligiannis N, Skaltsounis L, Ganzera MJPm, (2015) Inhibition of collagenase by mycosporine-like amino acids from marine sources. Planta Med. 81(10):813-820

47. El-Hady FKA, Abdel-Aziz MS, Shaker KH, El-Shahid ZAJIJPSRR (2014) Tyrosinase, acetylcholinesterase inhibitory potential, antioxidant and antimicrobial activities of sponge derived fungi with correlation to their GC/MS analysis. 26 (2):338-345

48. Rescigno A, Sollai F, Pisu B, Rinaldi A, Sanjust EJJoEl, Chemistry $M,(2002)$ Tyrosinase inhibition: general and applied aspects. J Enzyme Inhib Med Chem. 17(4):207-218

49. Zhang H, Zhao Z, Wang HJMd (2017) Cytotoxic natural products from marine sponge-derived microorganisms. Mar. Drugs. 15(3):68

50. Mehbub MF, Lei J, Franco C, Zhang WJMd (2014) Marine sponge derived natural products between 2001 and 2010: trends and opportunities for discovery of bioactives. Mar. Drugs. 12(8):4539-4577

51. El-Mesallamy AM, Hussein SA, Gerby ME (2013) El Azim MHAJNPAIJ. Phenolic composition and biological activities of 
methanolic extract of strawberry leaves (Fragaria ananassa) 9:251-265

52. El-Mousallamy AM, Hussein SA, Merfort I, Nawwar MAJP (2000) Unusual phenolic glycosides from Cotoneaster orbicularis 53(6):699-704

53. Yamamoto N, Moon J-H, Tsushida T, Nagao A, Terao JJAoB, Biophysics (1999) Inhibitory effect of quercetin metabolites and their related derivatives on copper ion-induced lipid peroxidation in human low-density lipoprotein. 372 (2):347-354

54. Kim HY, Moon BH, Lee HJ, Choi DHJJoE, (2004) Flavonol glycosides from the leaves of Eucommia ulmoides $\mathrm{O}$ with glycation inhibitory activity. Nutrients. 93(2-3):227-230

55. Kong C-S, Seo YJI, immunotoxicology, (2012) Antiadipogenic activity of isohamnetin 3-O- $\beta$-D-glucopyranoside from Salicornia herbacea. Immunopharmacol Immunotoxicol. 34(6):907-911

56. Bv K, Van den Berg A, Van Ufford HQ, Van Dijk H, Labadie RJPm, (1992) Anti-inflammatory activity of gallic acid. Planta Med. 58(06):499-504

57. Mukherjee G, Sachan A, Ghosh S, Mitra AJTJog, microbiology a, (2006) Conversion of sinapic acid to syringic acid by a filamentous fungus Paecilomyces variotii. The Journal of General and Applied Microbiology. 52(2):131-135
58. Xie X-G, Huang C-Y, Fu W-Q, Dai C-CJFb (2016) Potential of endophytic fungus Phomopsis liquidambari for transformation and degradation of recalcitrant pollutant sinapic acid. 120 (3):402-413

59. Riaz A, Rasul A, Hussain G, Zahoor MK, Jabeen F, Subhani Z, Younis T, Ali M, Sarfraz I, Selamoglu Z (2018) Astragalin: A Bioactive Phytochemical with Potential Therapeutic Activities. Adv Pharmacol Sci 2018:9794625-9794625. https://doi. org/10.1155/2018/9794625

60. Su T-R, Lin J-J, Tsai C-C, Huang T-K, Yang Z-Y, Wu M-O, Zheng Y-Q, Su C-C, Wu Y-J (2013) Inhibition of melanogenesis by gallic acid: possible involvement of the PI3K/Akt, MEK/ERK and Wnt/ $\beta$-catenin signaling pathways in B16F10 cells. Int J Mol Sci 14(10):20443-20458. https://doi.org/10.3390/ijms141020443

Publisher's Note Springer Nature remains neutral with regard to jurisdictional claims in published maps and institutional affiliations. 